Health and Medicine | Professor Tetsuya Kodama

\section{Lymph nodes targeted in novel cancer therapy}

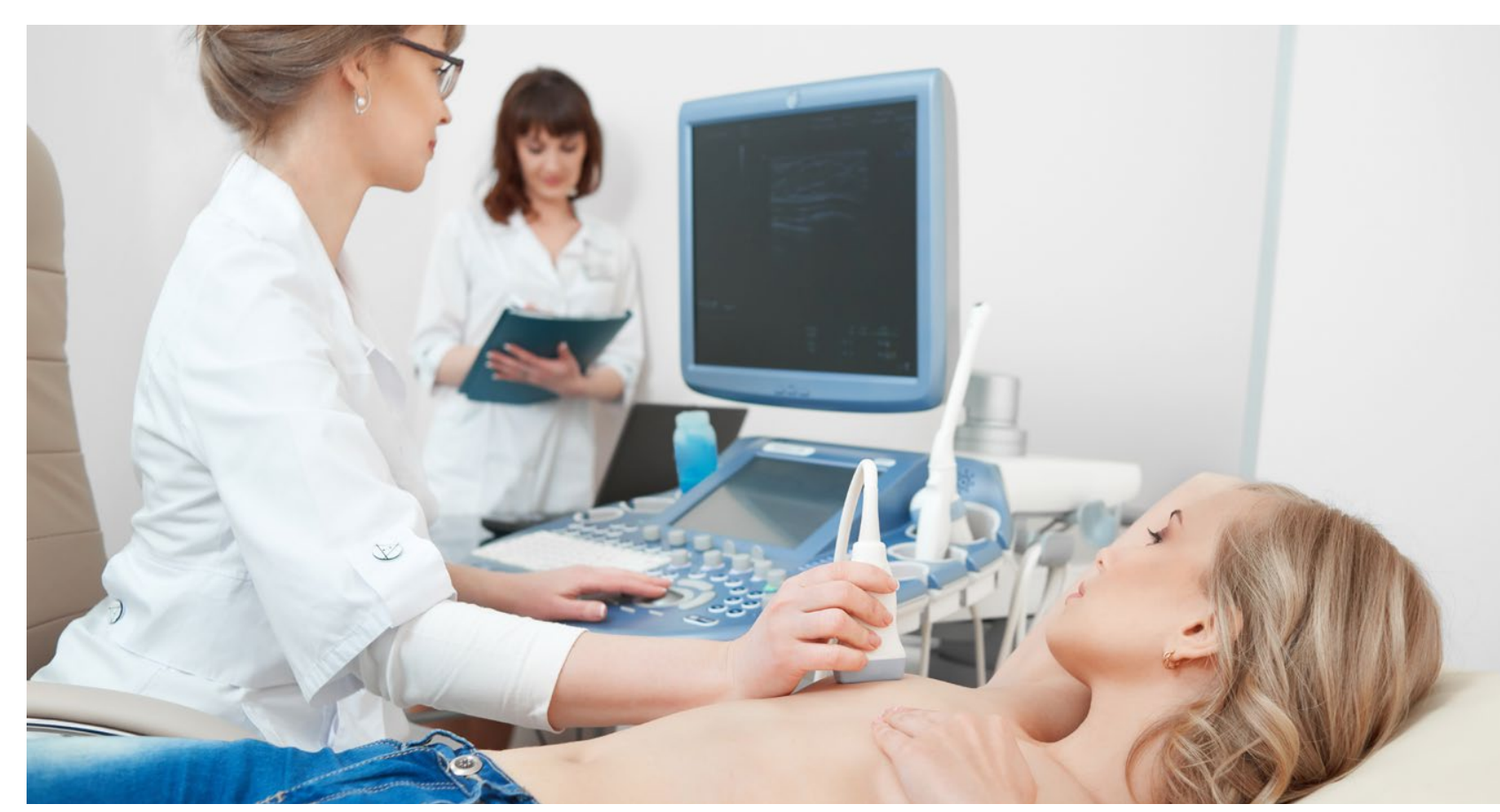

$90 \%$ of global deaths caused by cancer are the result of its metastasis. Cancer can spread throughout the body through the blood system or funding from ISPS KAKENH Professor Tetsuya Kodama at Tohoku University in Japan and his collaborators established a novel mouse model to study cancer metastasis through the lymphatic system. With this model, they identified lymph node metastasis as the starting point of metastatic disease. on developing a new cancer therapy to target specifically lymph node metastasis and holds huge revolutionary potential for the treatme of metastatic cancer.

\begin{abstract}
ancer was estimated to have caused 9.6 million deaths globally in $2018 . \ln 90 \%$ of cases, it is not the primary tumour that is the sprotis mastasis. Metastasis cells around the body resulting in the growth of secondary, metastatic tumours. When a tumour metastasises, it can spread through the bloodstream (known as a hematogenous spread)

metastasise via the lymphatic system: these cancer cells travel to nearby lymp where they des regional lymph nodes) where they develop, causing the lymph around the body to other'downstreat' lymph nodes. When cancer has reached the lymph nodes, it is also possible cancer will spread into the bloodstream in what has been termed 'lymph nodemediated hematogenous metastasis'.
\end{abstract}

In $90 \%$ of cases, it is not the primary tumour that is lethal, but it's metastasis.

or through the lymphatic system (known as a lymphatic spread). The lymphatic that circulate lymph (fluid containing white blood cells, amongst other components), forming an important part of the body's fin hu system. Cancers the sentinel lymp node/nodes) is the 'start point' of metastasis. Professor Tetsuya Kodama to effectively treat metastatic cancer system is comprised of lymphatic vesse by targeting these lymph nodes with the lymphatic system.

Staging of cancer is based on TNM classification. Tcategory describes the primary tumour site. N category describes the regional lymph node involvement. M category describes the presence or otherwise of distant metastatic spread. In TNM classification, TO is defined as no detection of a primary tumour, $\mathrm{NO}$ is the absence of metastasis in the regional lymph nodes, and MO is defined as no distant metastases. The factors of TNM classification other than $\mathrm{TO}, \mathrm{NO}$ or $\mathrm{MO}$ are defined by the type of tumour. Classifying tumours according to the TNM system, however, has its limitations. One such limitation is the frequency at which lymph If cancer has spread beyond the sentinel. lymph node, but the downstream lymph nodes do not exhibit noticeable swelling 'false-negative' results are obtained This makes the conventional treatments (surgical lymph node resection, radiotherapy and/or chemotherapy) for lymph node metastasis unsuitable to treat No false-negative metastatic cancer.

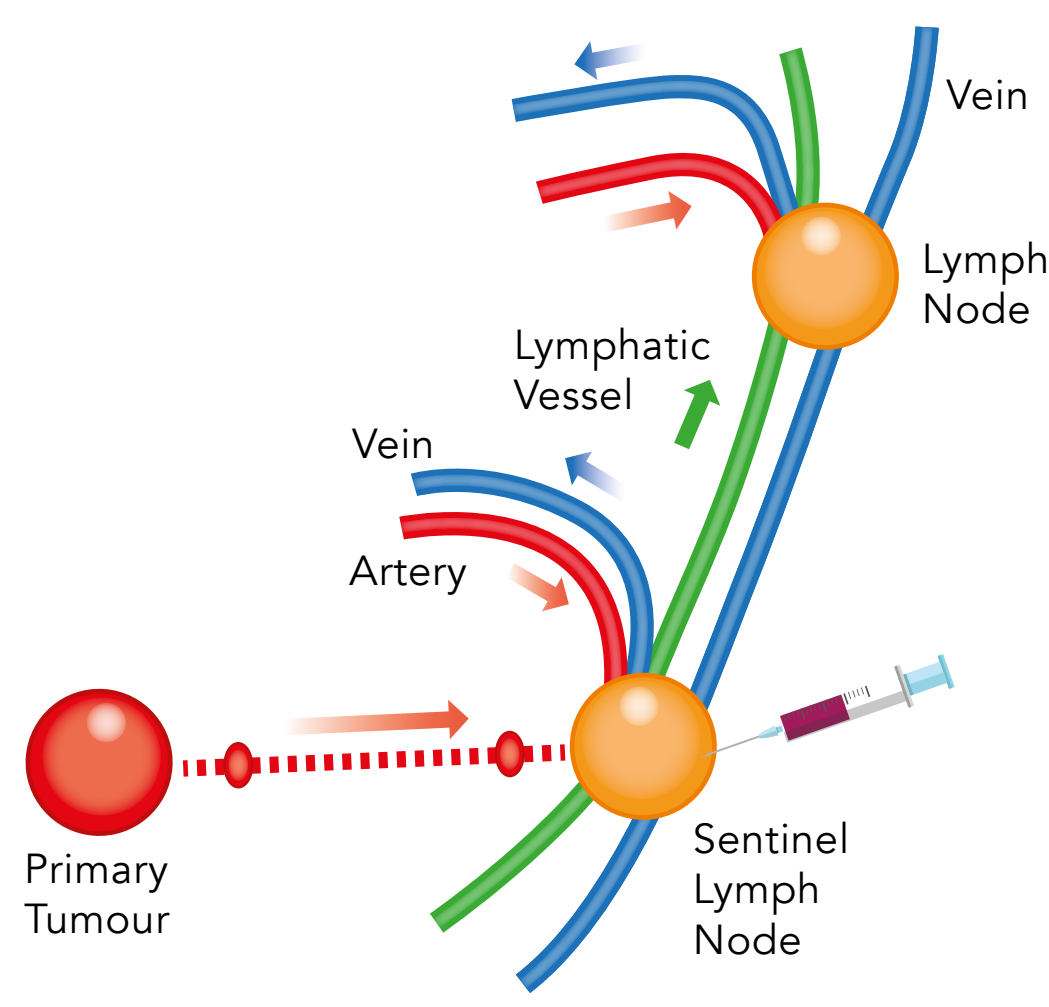

LYMPHATICS DRUG DELIVERY SYSTEM

A high dose of an anticancer drug into a metastatic lymph node (sentinel lymph node) and deliver the drug to downstream lymph nodes Iymphatic network, thereby treating or pre
injected lymph node and downstream lymph nodes. The amount of drug used in the LDDS is extremely Chemotherapy can also cause adverse cor
In addition, chemotherapy administered into the bloodstream through an I.V. injection or infusion (known as systemic administration) has been found to have limited success in treating cancer in the lymphatic system as the chemotherapy

drugs become diluted in the blood resulting in low concentrations of the drug reaching the lymph nodes. side effects because it circulates in the body, rather than selectively treating th malignant target.

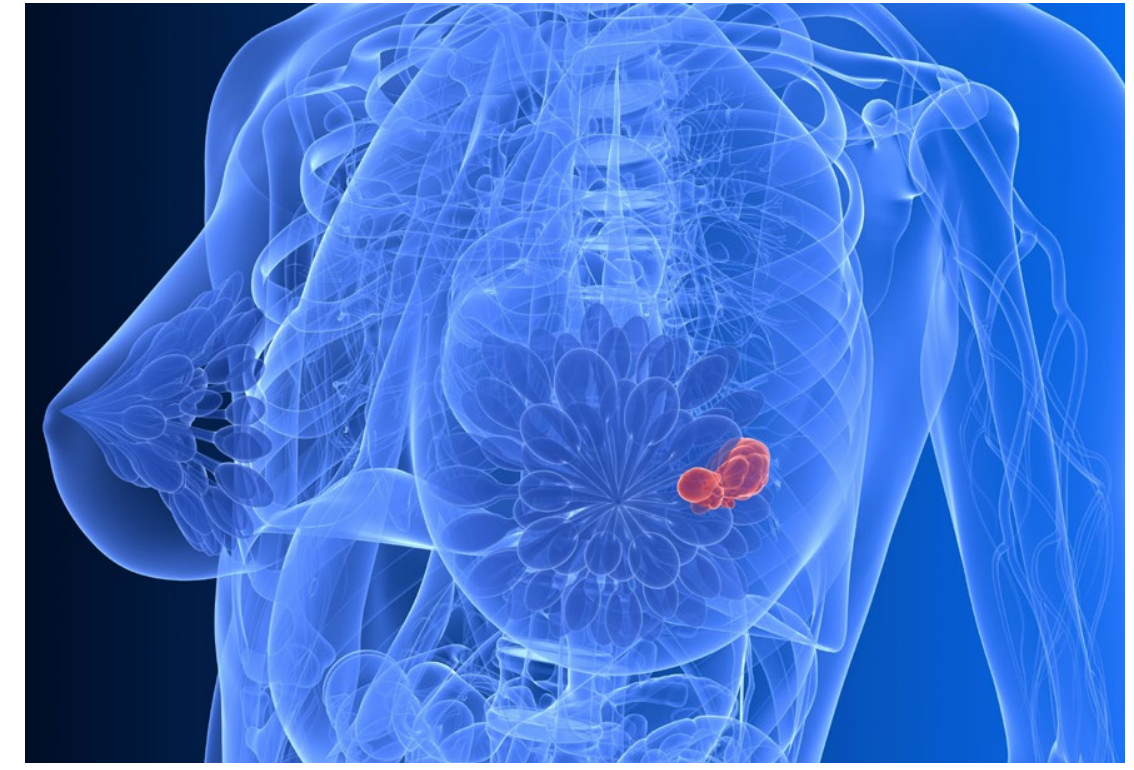

When you go to the hospital feeling abnormality such as a lump, the palpation is performed first. Next,
image diagnosis such has mammography, ulltrasonography (echo) MRl and CT are conducted, finally
biopsyis sond Research into in of lymph node metastasis had been previously limited largely due to a lack disease. Kodama and his collaborators mode to establish a novel mouse model that contains lymph nodes of a similar size to that of humans (up to 10 to be studied in animals for the first time of drugs to the lymphatic system

through injections in lymph nodes (also known as intranodal injections). Using fuorescent dyes, Kodama and his team were able to visualise how fluid drains ' novel mstream 'lymph nodes in their mphatic drug delivery systems were able to be developed to specifically target cancer in the lymphatic system.

Using their mouse model, Kodama and his collaborators observed how decreased flow from the senting
Lymphatic drug delivery systems were cancer metastasises through the 


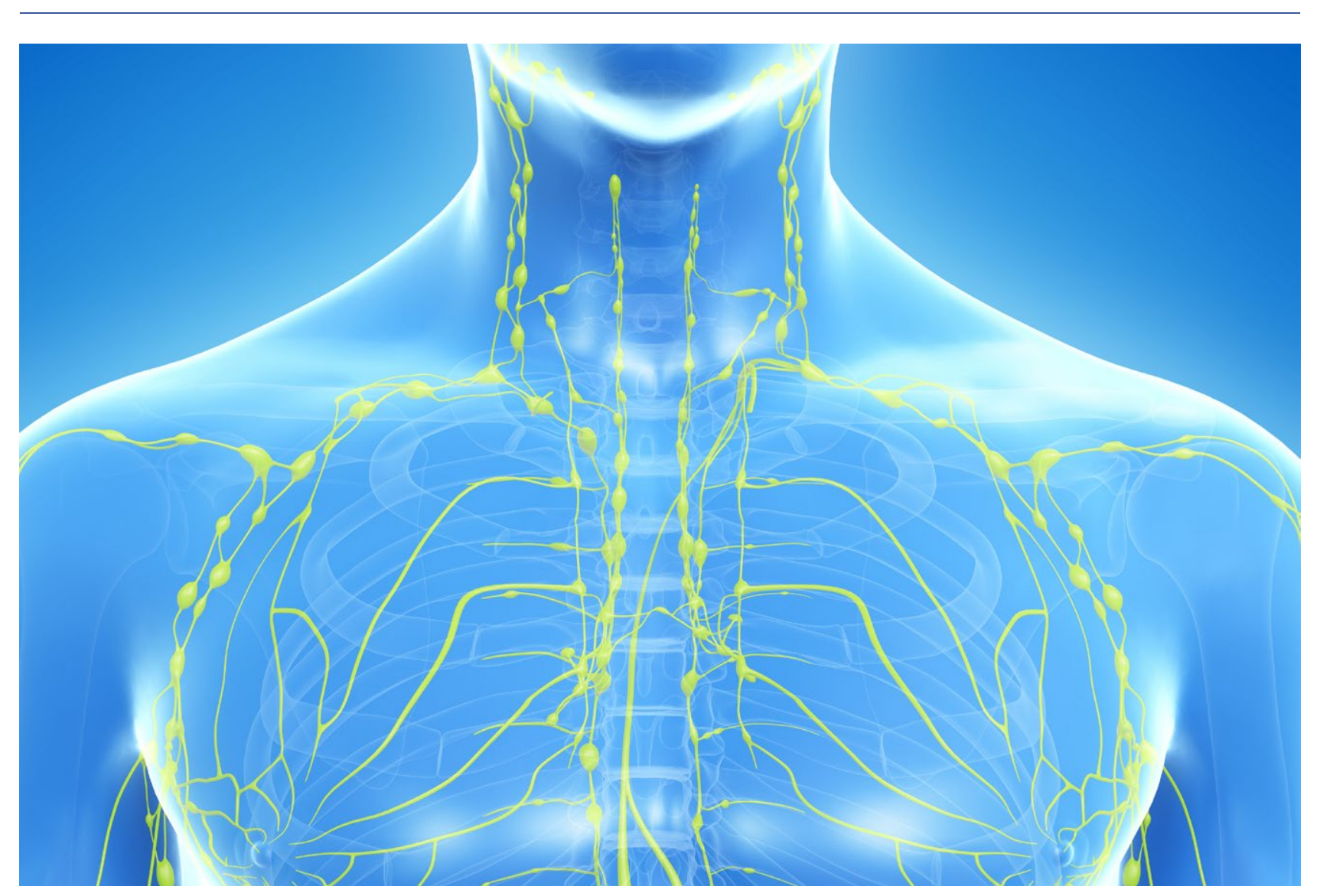

The lymphatic network consists of lymphatic vessels
total $600-800$ lymph nodes spread out in the body.

lymph node to downstream lymph nodes when cancerous tumours develop
in the vessels between them. Instead, the in the vessels between them. Instead, the
flow was orientated to the blood system. flow was orientated to the blood system. This observation led the researchers
to theorise that 'lymph node-mediated to theorise that 'lymph node-mediated
hematogenous metastasis' is the hematogenous metastasis' is the final stage in lymph node metastasis, and the sentinel lymph node should, of cancer metastasis.

Cisplatin, a chemotherapy drug used to treat cancers such as breast cancer and head and neck cancer, injected into the sentinel lymph node of mice was in false-negative metastatic lymph nodes. In addition, Kodama and his team observed fewer adverse side effects to cisplatin, such as kidney disease, when given as an intranodal injection than when given systemically through an I.V. By administering chemotherapy with lymphatic drug delivery systems, the concentration of the drug is much higher when it reaches the target lymph nodes, meaning dosage can be much Chemotherapy can be injected and
This treatment has a huge potential to reduce cancer mortality by stopping cancer before it can spread beyond the lymphatic system.

allowed to flow to downstream lymph chemotherapy to ensure the drug flowed nodes prior to surgical resection of the recurrence This treatmenthas a huge potential to reduce cancer mortality by stopping cancer before it can spread beyond the lymphatic system.

Kodama and his colleagues are currently researching factors that could limit the success of lymphatic drug delivery systems in treating false-negative lymph node metastasis. Their most recent paper describes an investigation into the impact of chemotherapy drug injection on the flow of the drug to downstream that factors such as injection pressure range of injection rates to administer the trial to investigate the safety and effectiveness of lymphatic drug delivery years, followed by Phase ll clinical trials to confirm efficacy.

Kodama and his colleagues' work has paved a new way in not only esearching lymph node metastasis using mice and thinking about how cancer metastasis, but also in how cancer delivery systems using chemotherapy could dramatically improve the survival rates across many types of cancer whilst The wast brefly discused of treatment. The work briefly discussed here is likely to revolutionise cancer therapy. primary tumour and sentinel lymph node, lymph nodes in mice. Their results show and the volume of the sentinel lymph node do not impact on the success of the The group will design Phase I clinical system using chemotherapy within a few therapy can be targeted. Lymphatic drug chemotherapy to ensure the drug flowed
JSPS KAKENHI Grant Number: 17K20077, 17H00865,

26242051, 24650286, 23300183, 21650124

\section{Collaborators}

Dr Shiro Mori, Tohoku University Hospital, Japan. - Dr Koichi Ito, Tohoku University Graduate School of Information Sciences.

- Professor Kiyoto Shiga, Iwate Medical School, Japan.

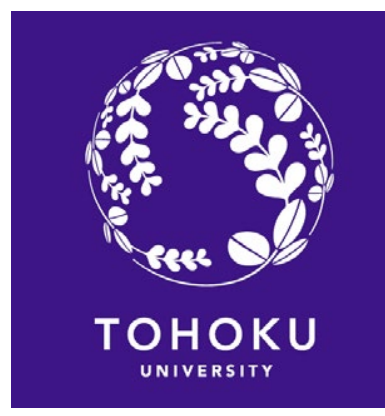

\section{Behind the Research}

\section{Professor Tetsuya Kodama}

Research Objectives

Metastasis, a characteristic of many tumour types, is estimated to be responsible for the death of $90 \%$ of al patients with cancer. However, effective treatment of metastasis has not been established. In Professor Tetsuya Kodama's laboratory, they focus on early diagnosis and treatment oflymph node metastasis, observed in most

\section{Detail}

Director of Biomedical Engineering Cancer Research Center Edical Engineering for Cancer Graduate School of Biomedical Engineering Tohoku

-1 Seiryo, Aoba, Sendai 980-8575, Japan

Bio

Dersuya Kodama is a Professor at Tohoku University. He is he Director of the Biomedical Engineering Cancer Research Center, Graduate School of Biomedical Engineering Tohok Chiversity. He gained a PhD in engineering and a PhD in medivity (Japan) in 1992 and 2011 Dr

\section{References}

Cancer Research UK (2017) TNM staging. [Online] Available at: https://www.cancerresearchuk.org/about-cancer// breast-cancer/stages-types-grades/tnm-staging [Accessed 17/10/2018]

Fujii, H., Horie, S., Takeda, K., Mori, S. and Kodama, T. (2018) 'Optimal range of injection rates for a lymphatic drug delivery system. Joumal or biophotonics, lonline] p.e201700401. Accessed 12/10/2018] -

Kodama, T., Hatakeyama, Y., Kato, S. and Mori, S. (2015). Visualization of fluid drainage pathways in lymphatic vessels and lymph nodes using a mouse model to test a lymphatic drug delivery system'. Biomedical optics express, [online] 6(1), pp.124-134. Available at: https://doi.org/10.1364/ BOE.6.000124 [Accessed 13/10/2018].

Kodama, T., Matsuki, D., Tada, A., Takeda, K. and Mori, S. (2016). 'New concept for the prevention and treatment of metastatic lymph nodes using chemotherapy administered via the lymphatic network'. Scientific reports, lonline] 6, p.32506. Available at: https://doi.org/10.1038/srep32506 [Accessed 13/10/2018

Tada, A., Horie, S., Mori, S. and Kodama, T. (2017). Therapeutic effect of cisplatin given with a lymphatic drug delivery system on false negative metastatic lymph nodes'. Htps://doiorg/101111/cas 13387 [Accessed 13/10/2018].

\section{Personal Response}

Cancer death rates are so high due to late diagnosis lymphatic drug delivery syst
mortality rates of cancer?

II Although our method targets NO, lymphatic drug delivery systems are applicable to lymph nodes ${ }^{3} \mathrm{NO}$, i.e, patients whose cancer has progressed into the lymphatic to patients who are unsuitable for surgical resection of their lymph nodes, due to factors such as age or if the cancer has region of lymph node dissection. For patients such as our method can improve life prognosis greatly. 